Check for updates

Cite this: Chem. Commun., 2020, 56,9497

Received 11th June 2020

Accepted 9th July 2020

DOI: $10.1039 / \mathrm{d} 0 \mathrm{cc} 04094 \mathrm{k}$

rsc.li/chemcomm

\section{Chiral molecular conductor with an insulator- metal transition close to room temperature $\uparrow$}

\author{
Jonathan I. Short, ${ }^{a}$ Toby J. Blundell, (D) ${ }^{a}$ Sara J. Krivickas, ${ }^{a}$ Songjie Yang, ${ }^{a}$ \\ John D. Wallis, (D) ${ }^{a}$ Hiroki Akutsu, ${ }^{b}$ Yasuhiro Nakazawa ${ }^{b}$ and Lee Martin (D) *a
}

\begin{abstract}
Materials exhibiting both chirality and conductivity do not exist in nature and very few examples have been synthesised. We report here the synthesis of a chiral molecular metal which remains metallic down to at least $4.2 \mathrm{~K}$. This material also exhibits roomtemperature switching capabilities with a transition upon cooling below $10{ }^{\circ} \mathrm{C}$.
\end{abstract}

Bulk chiral molecular conductors are highly sought after because they can help formulate a theoretical model for electron conduction in chiral materials by exhibiting electrical magneto-chiral anisotropy (eMChA). The first observation of the effect of chirality upon superconductivity was recently observed in an individual nanotube of tungsten disulphide which shows asymmetric electron transport in an applied magnetic field. ${ }^{1}$ Differences in resistivity in applied magnetic fields between opposing enantiomers of the same material (eMChA) have been observed in bismuth helices, ${ }^{2}$ and carbon nanotubes. ${ }^{3}$ However, the lack of suitable enantiopure materials has made it difficult to formulate a theoretical model for electron conduction in bulk chiral conductors. The synthesis of radical-cation salts from enantiopure molecules (organosulfur donors, anions and/or solvents) provides an excellent opportunity to study eMChA in a chiral lattice which also shows conductivity or superconductivity. Radical-cation salts have been synthesized from a variety of chiral or racemic anions. ${ }^{4}$ The spatial arrangement of opposing $\Delta$ and $\Lambda$ enantiomers of tris(oxalato)metallates in the anion layers determines the packing arrangement of the BEDT-TTFs in the donor layer which in

\footnotetext{
${ }^{a}$ School of Science and Technology, Nottingham Trent University, Clifton Lane, Nottingham, NG11 8NS, UK

${ }^{b}$ Department of Chemistry, Graduate School of Science, Osaka University,

1-1 Machikaneyama, Toyonaka, Osaka 560-0043, Japan

$\dagger$ This article is dedicated to Professor Peter Day. LM and TB would like to thank the Royal Society and Leverhulme Trust for financial support (LT170022 and RPG2019-242).

\# Electronic supplementary information (ESI) available: Fig. S1-S3. CCDC 1984873 and 1984874. For ESI and crystallographic data in CIF or other electronic format see DOI: 10.1039/d0cc04094k
}

turn determines the conducting behaviour. ${ }^{5}$ Using a chiral solvent for electrochemical growth of these salts can lead to a chiral guest molecule being included in the lattice, ${ }^{6}$ or to chiral induction producing a chiral salt which contains only a single enantiomer of tris(oxalato)metallate. ${ }^{7}$

The most successful method for producing chiral radicalcation salts has been by using enantiopure donors based on EDT-TTF $^{8}$ or BEDT-TTF. ${ }^{9}$ Whilst still difficult to obtain highly crystalline materials, the donor tetramethyl-BEDT-TTF has produced several enantiopure salts. ${ }^{10}$ eMChA has been observed in salts of enantiopure DM-EDT-TTF with $\mathrm{ClO}_{4}$ which crystallise in enantiomorphic space groups $P 6_{2} 22$ and $P 6_{4} 22$, and show metallic behaviour above $40 \mathrm{~K}^{11}$ Enantiopure DM-EDT-TTF also gives semiconducting salts with $\mathrm{PF}_{6}$ whilst the racemate adopts a different packing arrangement and shows metallic behaviour. ${ }^{12}$ Despite this seminal discovery, these are still the only materials of this type synthesised to date and more bulk chiral conducting materials are required to fully understand this phenomenon of eMChA.

We have previously reported the synthesis of the novel chiral donor molecule $\left(1^{\prime} R, 5 S\right)-N$-( $1^{\prime}$-phenylethyl)(BEDT-TTF)acetamide 1 (Scheme 1) along with its diastereomer $\left(1^{\prime} R, 5 R\right) \cdot{ }^{13}$ This is one of only four monosubstituted BEDT-TTFs which have been prepared as a pure enantiomer. ${ }^{14}$ This BEDT-TTF is also novel in having a side-chain with an aromatic ring with potential to pack with planar acceptor molecules. We report here the first radicalcation salt of $\mathbf{1}$ which is a chiral conductor showing metallic behaviour down to $4.2 \mathrm{~K}$. Most notably, this salt also undergoes an insulator to metal transition (IM) close to room temperature. Materials which are able to switch their properties depending on

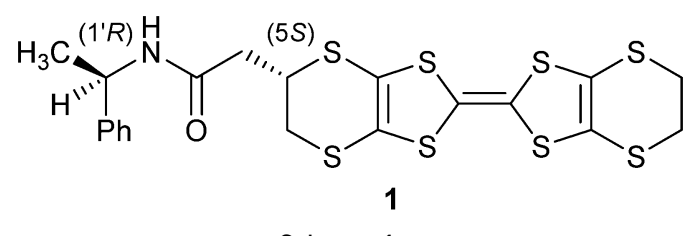

Scheme 1 
their temperature could lead to a new generation of electronic devices. Atom-by-atom changes to this material offers the potential for the synthesis of a family of materials each with a different IM transition temperature.

$\theta-(\mathbf{1})_{4}$ TCNQ crystallises in a monoclinic crystal system in chiral space group $C 2$ at $150 \mathrm{~K}$, but in a triclinic system in space group $P 1$ at $300 \mathrm{~K}$ with the $a$ axis being halved. $\S$ At $150 \mathrm{~K}$ the asymmetric unit consists of one donor molecule and a quarter of a TCNQ molecule, whilst at $300 \mathrm{~K}$ there is somewhat unusual disorder. In the donor stacking direction (along the $b$-axis) there are two donors with occupancies of 0.87 and 0.13 , respectively. Both donors are too close to co-exist, since the $b$-axis length is only $4.8570(5) \AA$. The minor occupancy donor was fixed to have the same shape as that of the major donor. One possibility is that one donor stack is slipped along the $b$-axis compared to the other. Such disorder is commonly observed at room temperature where the occupancy of both donors is 0.5. Another possibility is the existence of a superstructure ${ }^{15}$ which will be discussed later.

In both the $150 \mathrm{~K}$ and $300 \mathrm{~K}$ phases the thick anion sheet and the donor layer stack alternately along the $a$ axis (Fig. 1) with the donors adopting an $\theta$-type packing (Fig. 2). Several BEDT-TTF salts with $\theta$-type packing are known, having monoclinic $C 2$ space group at low temperature and orthorhombic I222 at room temperature. Mori et al. ${ }^{16}$ have previously shown a relationship between the dihedral angle between neighbouring donor stacks and the physical properties of the $\theta$ salts involving the series $\theta$-(BEDT-TTF $)_{2} \mathrm{MM}^{\prime}(\mathrm{SCN})_{4}\left(\mathrm{M}=\mathrm{Tl}, \mathrm{Rb}, \mathrm{Cs}, \mathrm{M}^{\prime}=\mathrm{Co}\right.$, $\mathrm{Zn})$. The ground state of these quarter-filled salts varies from insulating to metallic with decreasing dihedral angle. The metal-insulator transition temperature in this series of $\theta$ salts varies from $250 \mathrm{~K}$ down to $20 \mathrm{~K}$ as the dihedral angle changes from $\sim 116^{\circ}$ to $105^{\circ} . \theta-(\mathrm{BEDT}-\mathrm{TTF})_{2} \mathrm{CsZn}(\mathrm{SCN})_{4}$ has a dihedral angle of $105^{\circ}$ and undergoes a metal-insulator transition at $20 \mathrm{~K}$, whilst $\theta$-(BEDT-TTF $)_{2} \mathrm{I}_{3}$ has a dihedral angle of $99^{\circ}$ and shows ambient pressure superconductivity at $\sim 4 \mathrm{~K}^{17}$

SQUID magnetometry on $\theta-(\mathbf{1})_{4}$ TCNQ showed no superconductivity down to $1.8 \mathrm{~K}$. Four-probe DC transport measurements were made on four single crystals of $\theta-(\mathbf{1})_{4}$ TCNQ and all

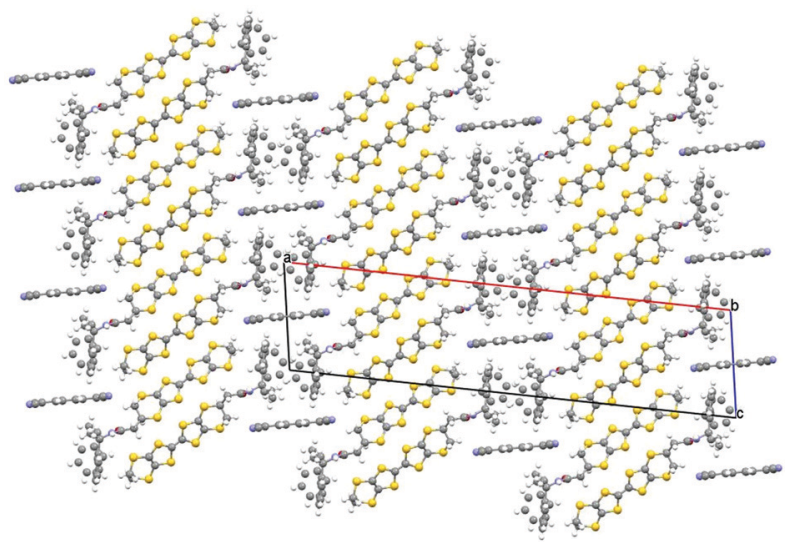

Fig. 1 Structure of $\theta-(1)_{4} T C N Q$ at $150 \mathrm{~K}$ viewed along the $b$ axis showing the disordered phenyl groups and TCNQ molecules.

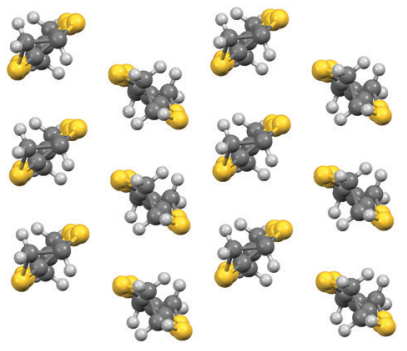

Fig. $2 \theta$-type packing of donor molecules.

showed metallic behaviour down to $4.2 \mathrm{~K}$. However, in contrast with other $\theta$ salts we observe in $\theta-(\mathbf{1})_{4}$ TCNQ both hysteresis and an insulator-metal transition (IM) at 285-290 K (Fig. 3). The following paragraphs discuss the structural features which lead to this IM transition.

Raman spectroscopy of $\theta-(\mathbf{1})_{4}$ TCNQ at $300 \mathrm{~K}$ shows only peaks corresponding to TCNQ ${ }^{-\bullet}\left(1387 \mathrm{~cm}^{-1}\right.$ and $\left.1613 \mathrm{~cm}^{-1}\right)$, whilst cooling below the IM transition temperature leads to the appearance of additional peaks corresponding to $\mathrm{TCNQ}^{0}$ (1450 $\mathrm{cm}^{-1}$ and $1600 \mathrm{~cm}^{-1}$ ). This change in TCNQ charge is reversible upon repeated heating and cooling cycles. SQUID magnetometry also confirms the transfer of charge from TCNQ to donor below the IM transition temperature.

In the crystal structure solution at $150 \mathrm{~K}$ the $-\mathrm{CH}\left(\mathrm{CH}_{3}\right)$ phenyl group on the donor side chain appears disordered over two positions, with pairs of these side chains from different donor stacks butted up against each other within the TCNQ layer (Fig. 1). We interpret the disorder in the TCNQ stack along the $c$ direction as the two different arrangements of these two neighbouring side chains, Ph1 and Ph2, alternating in the order -Ph1-Ph2-TCNQPh1-Ph2-TCNQ- so only every other TCNQ is present (Fig. 4). The stacks along $c$ above and below in the $b$ direction, will be such that the TCNQs lie above the block of four side chains. The short $b$ axis cannot accommodate adjacent TCNQs. This pattern is not in sync for all parallel layers, hence leading to the apparent disorder.

The hysteresis of the resistivity curves indicates that the transition is a first-order transition. The salt is a stable metal from $250-4.2 \mathrm{~K}$ therefore at least from 150 to $2 \mathrm{~K}$ the donor layer should have a Pauli contribution to the magnetism which usually has no temperature dependence. Therefore, we fitted the magnetic data in a $\chi^{T}$ graph using a Curie-Weiss model

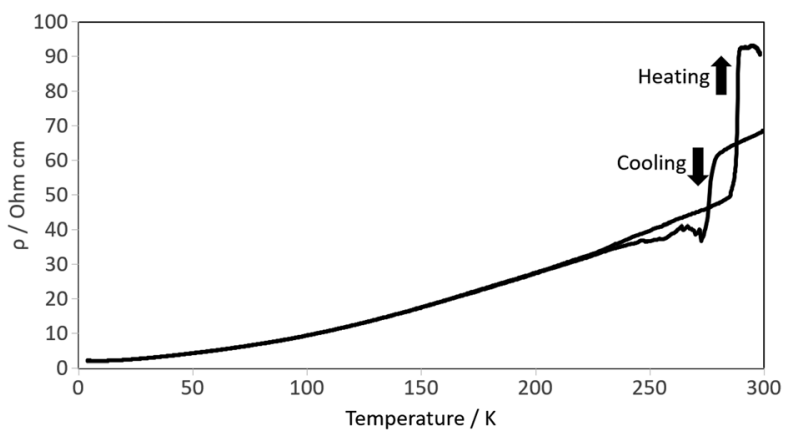

Fig. 3 Electrical resistivity measured on a single crystal of $\theta-(\mathbf{1})_{4} \mathrm{TCNQ}$. 


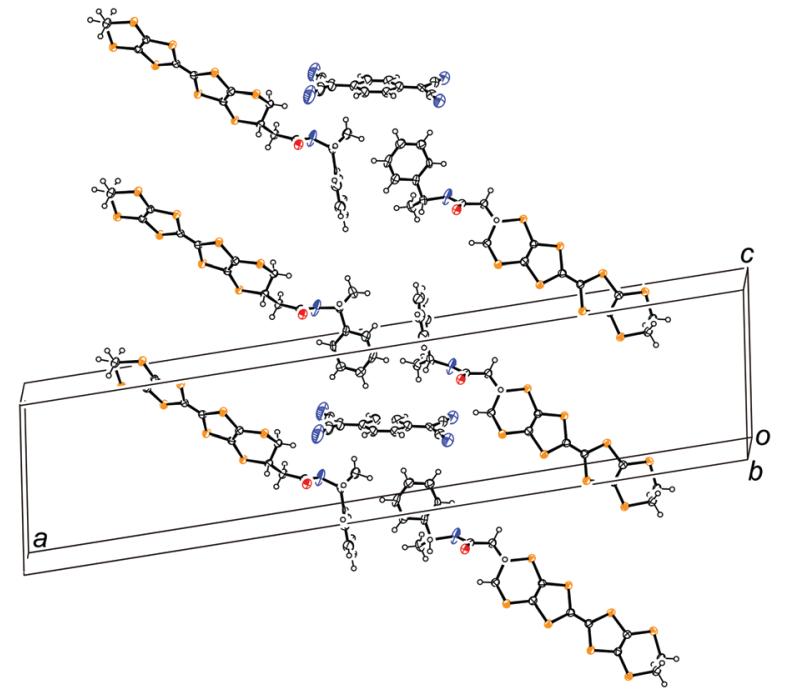

Fig. 4 Structure of $\theta-(\mathbf{1})_{4} \mathrm{TCNQ}$ at $150 \mathrm{~K}$ viewed along the $b$ axis showing one of the orientations of the disordered phenyl groups and TCNQ molecule.

with a temperature-independent factor $a(\chi=C /(T-\theta)+a)$ leading to values of $C=0.228 \mathrm{emu} \mathrm{K} \mathrm{mol}{ }^{-1}, \theta=-0.09 \mathrm{~K}$ and $a=7.7 \times 10^{-4} \mathrm{emu} \mathrm{mol}^{-1}$. The $C$ value and the Raman results $\left(\mathrm{TCNQ}^{0}\right.$ and $\mathrm{TCNQ}^{-}$co-exist) suggest that the average charge per TCNQ is found to be 0.61 , which means approximately $6 / 10$ TCNQ molecules are monoanionic and 4/10 are neutral in the lower temperature phase. The $\theta$ of almost zero indicates that the magnetic interactions between TCNQ monoanions are negligible, which suggests that the $\mathrm{TCNQ}^{\circ}$ and $\mathrm{TCNQ}^{-}$are almost alternately located in the TCNQ chain along the shortest $b$ axis. This is to be expected because contacts between monoanions would provide a large Coulomb repulsion. It raises a question as to why the TCNQ chain does not select complete alternation, namely $\mathrm{TCNQ}^{0}: \mathrm{TCNQ}^{-}=1: 1$ (the actual ratio is $2: 3$ ).

The donor is chiral so all $\mathrm{NH}-\mathrm{C}=\mathrm{O}$ groups are directed along the $b$ axis, the stacking direction, in the same sense - this means that the donor layer has a relatively large dipole moment $\sim 4$ Debye, and voltage $\sim 0.5-0.6 \mathrm{eV}$. As a result, TCNQ chains should cancel the donor's dipole moment. We speculate that the TCNQ monoanions' distribution is not equal but biased to cancel the dipole moments provided by the amide groups of the donor molecules, i.e. the NH side of the crystal includes more $\mathrm{TCNQ}^{-}$molecules than the opposite $\mathrm{C}=\mathrm{O}$ side of the crystal. Thus, the non-stoichiometric TCNQ charge is due to the donor chirality-induced dipole moment.

The temperature-independent factor $a$ is larger than that of a normal 2:1 salt (maybe double). However, the average donor charge of $0.15^{+}$in this $4: 1$ salt is unusual implying that a large $a$ value may be possible for such a unique situation. Next, we subtracted the $a$ value from the total magnetic susceptibility to produce the $\chi T-T$ plot in Fig. 5. This indicates that spin concentration decreases suddenly at about $270 \mathrm{~K}$ from 1.0 and becomes constant, $\approx 0.6$. This strongly indicates that a charge-change (CC) transition occurs from $\mathrm{TCNQ}^{-}$only to the mixture of $\mathrm{TCNQ}^{0}$ :TCNQ $^{-}=1: 1$. Neutral-Ionic (NI) transitions

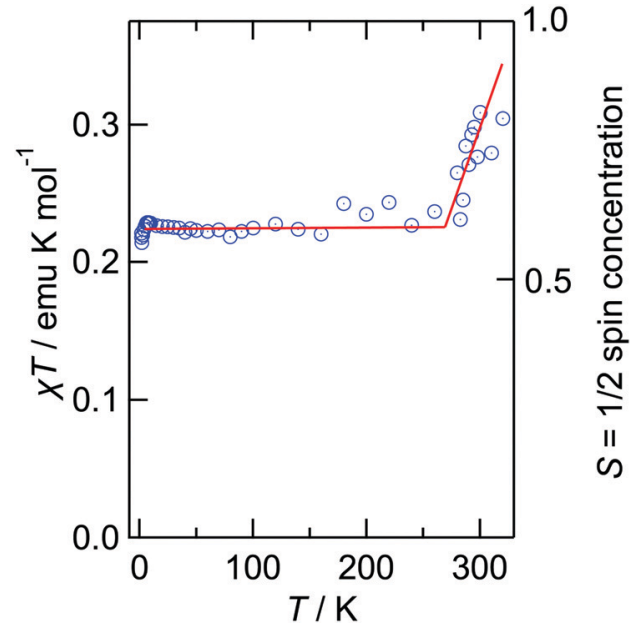

Fig. $5 \quad \chi^{T}$ versus $T$ for $\theta-(\mathbf{1})_{4} T C N Q$.

(e.g. TTF-chloranil) are very well known but this is the first example of a CC transition to the best of our knowledge. This implies that at $300 \mathrm{~K}$ relatively large thermal motion cannot cancel the imbalance of the dipole moments between the donor and TCNQ chains as discussed in detail later.

Fig. S1 (ESI ) shows the band calculations for $\theta-(\mathbf{1})_{4} \mathrm{TCNQ}$ at $300 \mathrm{~K}$ (top) and $150 \mathrm{~K}$ (bottom). The Fermi surfaces are simple at both temperatures indicating that the salt should be metallic. Owing to the incommensurate band filling of 0.92 at $150 \mathrm{~K}$ where $\mathrm{TCNQ}^{0.61-}$, it is impossible for them to become either band or Mott insulators. The $300 \mathrm{~K}$ case, which is in a commensurate insulating state, can be a Mott or a chargeordered state, but it is somewhat unusual because all TCNQs are monoanions, whose negative charges are uniformly distributed in the crystal at room temperature. Indeed, a chargeordered state should be observed at $150 \mathrm{~K}$, where neutral and monoanionic TCNQ co-exist, which provide a periodic or random potential, but it is indeed a stable metallic phase.

The Ewald sphere of the room temperature phase is shown in Fig. S2 (ESI $\$$ ), which indicates the existence of extra spots at $300 \mathrm{~K}$ at $h=0.5 n$ and $k=0.5 n$, where $n=$ some odd numbers. This indicates the formation of donor octamers. The crystallographic data at $300 \mathrm{~K}$ indicates that the phenyl ring on the chiral carbon has very large temperature factors, suggesting that the phenyl rings rotate above the IM transition. This leads to weaker donor-donor interactions, i.e. this provides negative chemical pressure to the donor layer. The unit cell expansion might disturb the conduction pathway and the resistivity therefore increases. If this disorder is an eight-fold superstructure (Fig. S3, ESI\$), the octamer has 2 holes which could explain why the salt is an insulator above the IM transition temperature, which is confirmed by the extra reflections on the Ewald sphere. We therefore speculate that the IM transition is a phenyl-ring motion-induced insulator-metal (IM) transition. Normally an insulating state (I) has lower energy than a metallic state (M), therefore the transition from $\mathrm{M}$ to $\mathrm{I}$ is normal, and the I to $\mathrm{M}$ transition is quite unusual. However, the insulating state in this case is caused by the negative pressure due to cell 
expansion of the almost free rotation of phenyl rings, which has large entropy ( $\approx R \ln 2$ ). Therefore, the insulating state can exist at a higher temperature than that of the metallic state. Below the IM transition temperature a charge-change (CC) transition occurs and the chirality-induced polarity leads to the TCNQ chains adopting non-stoichiometric charge to create the opposite polarity, causing the donor layer to be metallic.

Bulk chiral molecular conductors are highly sought after because they can help formulate a theoretical model for eMChA. We report the synthesis of a chiral molecular metal which remains metallic down to at least $4.2 \mathrm{~K}$. $\theta$ salts with BEDT-TTF $^{0.5+}$ invariably lose their metallic state at some temperature, but the BEDT-TTF ${ }^{0.15+}$ salt reported here keeps the metallic state down to very low temperatures. This opens the possibility of controlling the band filling as a way to reach superconductivity in $\theta$ salts. We are currently synthesising structurally related donor molecules, including the other three stereoisomers of this salt, and investigating other methods for synthesis of salts of this type.

This salt shows the first example of a charge-change (CC) transition to the best of our knowledge. Below the insulator-metal transition temperature the chirality-induced donor amide polarity leads to the TCNQ chains adopting non-stoichiometric charge to create the opposite polarity, causing the donor layer to be metallic.

This salt also exhibits room-temperature switching capabilities with a transition from the insulating to the metallic state upon cooling below $10{ }^{\circ} \mathrm{C}$. Materials which are able to switch their properties depending on their temperature could lead to a new generation of electronic devices. Atom-by-atom changes to this material offers the potential for the synthesis of a family of materials each with a different IM transition temperature.

\section{Conflicts of interest}

There are no conflicts to declare.

\section{Notes and references}

$\S 45.7 \mathrm{mg}$ of donor $\mathbf{1}$ and $17.6 \mathrm{mg}$ TCNQ in $13 \mathrm{ml}$ dichloromethane was heated under reflux for two hours whilst stirring. Slow evaporation gave black starbursts of crystalline needles of a $4: 1$ salt of donor 1 with TCNQ. Crystal data at $150 \mathrm{~K}:\left(\mathrm{C}_{20} \mathrm{H}_{19} \mathrm{~S}_{8} \mathrm{~N}_{1} \mathrm{O}_{1}\right)_{4} \cdot \mathrm{C}_{12} \mathrm{H}_{4} \mathrm{~N}_{4}, M=2387.62$, black needle, $a=46.8847(19) \AA, b=4.9361(2) \AA, c=11.2909(5) \AA, \beta=99.121(7)^{\circ}, U$ $=2580.0(2) \AA^{3}, T=150 \mathrm{~K}$, space group $C 2, Z=1, \mu=7.136 \mathrm{~mm}^{-1}$, reflections collected $=12105$, independent reflections $=5838, R_{1}=0.0442$, $\mathrm{w} R_{2}=0.1289\left[F^{2}>2 \sigma\left(F^{2}\right)\right], R_{1}=0.0515, \mathrm{w} R_{2}=0.1323$ (all data). CCDC 1984873. Crystal data at $300 \mathrm{~K}:\left(\mathrm{C}_{20} \mathrm{H}_{19} \mathrm{~S}_{8} \mathrm{~N}_{1} \mathrm{O}_{1}\right)_{2} \cdot 0.5\left(\mathrm{C}_{12} \mathrm{H}_{4} \mathrm{~N}_{4}\right), M=$ 1193.81, black needle, $a=23.723(2) \AA, b=4.8570(5) \AA, c=11.6968(10) \AA$, $\alpha=89.841(6)^{\circ}, \beta=81.637(6)^{\circ}, \gamma=87.168(6)^{\circ}, U=1331.8(2) \AA^{3}, T=300 \mathrm{~K}$, space group $P 1, Z=1, \mu=6.912 \mathrm{~mm}^{-1}$, reflections collected $=12845$, independent reflections $=9915, R_{1}=0.0997, \mathrm{w} R_{2}=0.2713\left[F^{2}>2 \sigma\left(F^{2}\right)\right]$, $R_{1}=0.1474, \mathrm{w} R_{2}=0.3145$ (all data). CCDC 1984874.

1 F. Qin, W. Shi, T. Ideue, M. Yoshida, A. Zak, R. Tenne, T. Kikitsu, D. Inoue, D. Hashizume and Y. Iwasa, Nat. Commun., 2017, 8, 1-6.
2 G. L. J. A. Rikken, J. Fölling and P. Wyder, Phys. Rev. Lett., 2001, 87, 236602; G. L. J. A. Rikken and E. Raupach, Nature, 1997, 390, 493-494.

3 V. Krstic, S. Roth, M. Burghard, K. Kern and G. L. J. A. Rikken, J. Chem. Phys., 2002, 117, 11315-11319; V. Krstić and G. L. J. A. Rikken, Chem. Phys. Lett., 2002, 364, 51-56; G. L. J. A. Rikken, Science, 2011, 331, 864-865.

4 M. Atzori, F. Pop, P. Auban-Senzier, C. J. Gómez-García, E. Canadell, F. Artizzu, A. Serpe, P. Deplano, N. Avarvari and M. L. Mercuri, Inorg. Chem., 2014, 53, 7028-7039; M. Atzori, F. Pop, P. Auban-Senzier, R. Clérac, E. Canadell, M. L. Mercuri and N. Avarvari, Inorg. Chem., 2015, 54, 3643-3653; J. R. Lopez, L. Martin, J. D. Wallis, H. Akutsu, Y. Nakazawa, J. I. Yamada, T. Kadoya, S. J. Coles and C. Wilson, Dalton Trans., 2016, 45, 9285-9293.

5 L. Martin, Coord. Chem. Rev., 2018, 376, 277-291.

6 L. Martin, P. Day, H. Akutsu, J. I. Yamada, S. Nakatsuji, W. Clegg, R. W. Harrington, P. N. Horton, M. B. Hursthouse, P. McMillan and S. Firth, CrystEngComm, 2007, 9, 865-867.

7 L. Martin, H. Akutsu, P. N. Horton and M. B. Hursthouse, CrystEngComm, 2015, 17, 2783-2790; L. Martin, H. Akutsu, P. N. Horton, M. B. Hursthouse, R. W. Harrington and W. Clegg, Eur. J. Inorg. Chem., 2015, 1865-1870.

8 N. Mroweh, F. Pop, C. Mézière, M. Allain, P. Auban-Senzier, N. Vanthuyne, P. Alemany, E. Canadell and N. Avarvari, Cryst. Growth Des., 2020, 20, 2516-2526; N. Mroweh, P. Auban-Senzier, N. Vanthuyne, E. Canadell and N. Avarvari, J. Mater. Chem. C, 2019, 7, 12664-12673; F. Pop, P. Auban-Senzier, E. Canadell and N. Avarvari, Chem. Commun., 2016, 52, 12438-12441.

9 S. Yang, F. Pop, C. Melan, A. C. Brooks, L. Martin, P. Horton, P. Auban-Senzier, G. L. J. A. Rikken, N. Avarvari and J. D. Wallis, CrystEngComm, 2014, 16, 3906-3916; S. Yang, A. C. Brooks, L. Martin, P. Day, M. Pilkington, W. Clegg, R. W. Harrington, L. Russo and J. D. Wallis, Tetrahedron, 2010, 66, 6977-6989; R. J. Brown, A. C. Brooks, J. P. Griffiths, B. Vital, P. Day and J. D. Wallis, Org. Biomol. Chem., 2007, 5, 3172-3182.

10 J. R. Galáln-Mascarós, E. Coronado, P. A. Goddard, J. Singleton, A. I. Coldea, J. D. Wallis, S. J. Coles and A. Alberolá, J. Am. Chem. Soc., 2010, 132, 9271-9273; F. Pop, S. Laroussi, T. Cauchy, C. J. Gomez-Garcia, J. D. Wallis and N. Avarvari, Chirality, 2013, 25, 466-474.

11 F. Pop, P. Auban-Senzier, E. Canadell, G. L. J. A. Rikken and N. Avarvari, Nat. Commun., 2014, 5, 1-6.

12 F. Pop, P. Auban-Senzier, A. Frạckowiak, K. Ptaszyński, I. Olejniczak, J. D. Wallis, E. Canadell and N. Avarvari, J. Am. Chem. Soc., 2013, 135, 17176-17186.

13 I. Awheda, S. J. Krivickas, S. Yang, L. Martin, M. A. Guziak, A. C. Brooks, F. Pelletier, M. Le Kerneau, P. Day, P. N. Horton, H. Akutsu and J. D. Wallis, Tetrahedron, 2013, 69, 8738-8750.

14 Q. Wang, P. Day, J. P. Griffiths, H. Nie and J. D. Wallis, New J. Chem., 2006, 30, 1790-1800; J. P. Griffiths, H. Nie, R. J. Brown, P. Day and J. D. Wallis, Org. Biomol. Chem., 2005, 3, 2155-2166; T. Ozturk, C. R. Rice and J. D. Wallis, J. Mater. Chem., 1995, 5, 1553-1556.

15 A. Kobayashi, T. Naito and H. Kobayashi, Synth. Met., 1995, 70, 1047-1048.

16 T. Mori, H. Mori and S. Tanaka, Bull. Chem. Soc. Jpn., 1999, 72, 179-197; H. Mori, S. Tanaka and T. Mori, Phys. Rev. B: Condens. Matter Mater. Phys., 1998, 57, 12023-12029; R. Kondo, M. Higa, S. Kagoshima, H. Hoshino, T. Mori and H. Mori, J. Phys. Soc. Jpn., DOI: $10.1143 /$ JPSJ.75.044716.

17 H. Kobayashi, R. Kato, A. Kobayashi, Y. Nishio, K. Kajita and W. Sasaki, Chem. Lett., 1986, 789-792; H. Kobayashi, R. Kato, A. Kobayashi, Y. Nishio, K. Kajita and W. Sasaki, Chem. Lett., 1986, 833-836; R. Kato, H. Kobayashi, A. Kobayashi, Y. Nishio, K. Kajita and W. Sasaki, Chem. Lett., 1986, 957-960; K. Kajita, Y. Nishio, S. Moriyama, W. Sasaki, R. Kato, H. Kobayashi and A. Kobayashi, Solid State Commun., 1987, 64, 1279-1284. 